\title{
Text Analysis and Knowledge Extraction
}

\author{
Fujio Nishida, Shinobu Takamatsu, \\ Tadaaki Tani and Hiroji Kusaka \\ Department of Electrical Engineering, \\ Faculty of Engineering, University of Osaka Prefecture, \\ Sakaj, Osaka, 591 JAPAN
}

\section{Introduction}

The study of text understanding and knowlegde extraction has been actively done by many researchers. The authors also studied a method of structured information extraction from texts without a global text analysis. The method is available for a comparatively short text such as a patent claim clause and an abstract of a technical paper.

This paper describes the outline of a method of knowledge extraction from a longer text which needs a global text analysis. The kinds of: texts agre expository texts ${ }^{2}$ or explanation texts Expository texts described here mean those which have various hierarchical headings such as a title, a heading of each section and sometimes an abstract. In this definition, most of texts, including technical papers reports and newspapers, are expository. Texts of this kind disclose the main knowledge in a top-down manner and show not only the location of an attribute value in a text but also several key points of the content. This property of expository texts contrasts with that of novels and stories in which an unexpected development of the plot is preferred.

This paper pays attention to such characteristics of expository texts and describes a method of analyzing texts by referring to information contained in the intersentential relations and the headings of texts and then extracting requested knowledge such as a summary from texts in an efficient way.

\section{Analysis of intersententiaj relations}

The global sentential analysis is performed

by using the information contained in the intersentential relations and the headings of a text by a method combining both the bottom-up and the top-down manner. Various kinds of intersentential relations, bave been proposed so far by many linguists ${ }^{12}$. By referring to these proposals, intersentential relations are classified tentatively into about 8 i.tens. They are a detail, an additionat, a parallel, a rephrase, an example, a temporal succession, a causal and a reasoning relation as described in the following subsections.

\section{(1) Detail relations}

If a term $t_{2}$ is the topic term in a sentence $\mathrm{S}_{2}$ and if $\mathrm{L}_{2}$ is a complementary term of the topic term $t$ i.n the preceding sentence $S$, as shown in Expr.(1), $S_{2}$ i.s called the detail of: $S_{1}$.

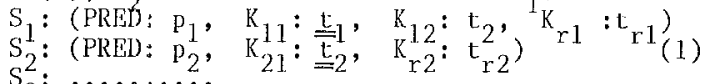

$\mathrm{S}_{3}^{2}: \ldots \ldots \ldots$

where $K: t$ represents a pair of a case label and a term, and the term with a double underline denotes a topic.

The sentence level of $S_{1}$ to that of $S_{2}$ depends on the property of the sentence $S_{3}^{2}$ following to $S_{2}$ and the relation among the terms

contained in the sentences $\mathrm{S}_{1} \mathrm{~S}_{2}$ and $\mathrm{S}_{3}$. If the sentence $S_{3}$ is connected to $S_{1}{ }_{1}$ more closely than $S_{2}$, for example, if the sentence $\mathrm{S}_{3}$ has the topic term $\mathrm{t}_{1}$ of the sentence $S_{1}$ as the topic, it is considered that the principal sentence is $S$ and the sentence level of $S_{2}$ is lower than that of $S_{\text {. }}$.

On the other hand, if $S_{1}$ is an introductory sentence of a term $t_{2}$ and the articles related to $t_{2}$ are described in some sentences following to $S_{1}$, or if $t_{2}$ is the global topic of the section, the sentence $S_{2}$ is considered the principal sentence. The global ${ }^{2}$ topic can be easily identified by inspecting the headings of the section the titile and the like, whatever it is an attribute name or an attribute value without reading through the whole text.

If the term $t_{2}$ in the sentence $S_{\text {. }}$ belongs to a kind of pronouns such as "in the following ones" or "as follows", the sentence $S_{2}$ is set at the same level as that of $S_{1} . \Lambda t$ the sumarization stage, the system tries to shorten the part consisting of $S_{1}$ and $S_{2}$ by replacing the pronoun $t_{2}$ in $S_{1}$ by the main content given in $S_{2}$, namely, the main part consisting of $\mathrm{t}_{\mathrm{r} 2}$ and $\mathrm{p}_{2}$.

[Example 1]

(a) $S_{1}$ : SGS receives an ordered triple from a user.

$\mathrm{S}_{2}^{1}$ : The triple's form is category, input-frames, conditions on the sentence.

$S_{3}$ : SGS regards the ordered triple as a goal.

$\mathrm{S}_{2}$ describes the content of a term "ordered trip.le" in $S_{1}$, and $S_{3}$ has the topic term "SGS" in $S_{1}$. Hence, $S_{2}$ is the detail of $S_{1}$, and $S_{1}$ is the princj.pal sentence.

(b) $S_{1}$ : In this section, the overview of Ll:G is described.

$S_{2}$ : LFG is an extension of context free grammar

and has the following two structures.

$\mathrm{S}_{3}$ : One is a c-structure which represents the surface word and phrase configurations, and the other is a f-structure......

S1. is an introductory sentence of a term "LFG" which is the global topic in a section taken from a text. $S_{2}$ has a kind of pronoun "the following two structures" whose contents are described in $S_{3}$. Hence, $S_{2}$ is the principal sentence and the sentence level of $\mathrm{S}_{2}$ is the same as that of $S_{3}$.

As a special case of detail relations, there are a rephrase relation and an example relation. T'hese intersentential relations between sentences $S_{1}$ and $\mathrm{S}_{2}$ can be identified by referring to their sentential constructions and sentence modj.fying adverbs such as "in other words" and "for example". The principal sentence of them is, in most cases, the sentence $S_{1}$ in an expository text.

(2) Addj.tional relations

If the current

sentential topic $t$ as sentences and describes sentence has the same that of the preceding another attributes or 
functions of the topic, the current sentence is called an additional sentence to the preceding sentences. The sentential form of the relation is

$$
\begin{aligned}
& \mathrm{S}_{1}:\left(\text { PRED }: \mathrm{p}_{1}, \mathrm{~K}_{\mathrm{i}}: \mathrm{t}_{1}, \mathrm{~K}_{1}: \mathrm{t}_{1}\right) \\
& \left.\mathrm{S}_{2}: \text { (PRED } \mathrm{p}_{2}, \mathrm{~K}^{\mathrm{t}}: \mathrm{K}_{1} \mathrm{~K}_{1}: \mathrm{t}_{2}\right)
\end{aligned}
$$

${ }^{2}$ The levels of both the sentences $S$ and $S_{2}$ are generally assumed to be the same except for the case that the global. topic is put in a predicate part of them. It can be also considered that additional relations hold among various sentential groups of the same level. such as chapters sections or paragraphs under a global topic contained in a ti.tle.

\section{(3) Other sentential relations}

There are other intersentential relations. They are roughly classified into a serial and a concurrent or an extended parallel relation.

A serial relation such as a temporal succession a causal or a reasoning relation has the same physical. location of focus or the same logical object while it has a time shift or a logical inference step shift between adjacent sentential groups.

A concurrent relation has the same time instant of the event occurrences or the sane stages of: logical inference while it has a distance or a spatial positional shift between the physical or the logica1 objects described i.n the adjacent sentential groups.

The level number of a sentence to the adjacent sentential. groups in these relations is assigned in a similar way to that of the detail or the additional relation by referring to the intersentential relations and the global topics.

In usual cases, the difference between a principa]. sentence level and the adjacent sentence level is usually set within one level.

As seen in the above, a sentence or a sentential group has an intersentential relation to some adjacent sentences or sentential groups. Tho intersentential relation between adjacent sentences j.s similar to a relation between adjacent: words or word groups combined through rewriting rules of a sentence. The intersentential relations are classified into two classes. One of them is a relation such as a detail relation which holds between a principal sentence and the auxiliary or modifying sentential group with a lower level. than the principal sentence as shown in Fig.l(a). "l'he other is a juxtaposition relation like an additional relation which holds among several coherent sentences with the same level in usual. as shown in Fig.l(b).

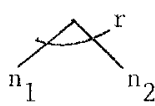

(a)

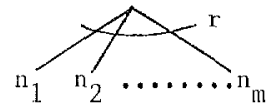

(b)
Fig.1 Intersentential relations

In these diagrams a leaf node represents a sentence of a text and an intermediate node denotes a representative sentence of the direct descendents or the princjpal parts of then. $A$ name $r$ attached to an arc bridging over several. branches denotes an intersentential relation.

\section{Text analysis}

An expository text has a ti.te and consists of several sections. The title shows the main topics of the text. The heading of each section shows local topics of each section and constitutes the attributes of the main topics.

Each of main sections sometimes has an introductory remark followed by the main part. T'he content of the main part is almost covered with the subframe predetermined by the heading and the title.

The global. cohesion of a section is assured by a relation jin which each majn part of the section shares some items of the same subframe wi.th other main parts.

Based on the above idea of text construction, a text analysis is done after parsing of each sentence. First, each pronoun is replaced by the antecedent noun word with the aids of an anaphora analysis. Then, the intermediate expression of each sentence of the text is transformed into the normal form in which each topic term is inherited together with a double underlined mark. The expressions to be normalized are object-apposition expressions, object-component expressions, predicate-cause expressions, expressions whick have a term consisting of a case label, and others .

After normalization, the part of topics and the content of each sentence are fi.rst: identified. Second, intersentential relations between two adjacent sentences are jdentified indeterministical.$1 y$ based on the assumptions of: two classes of intersentential relations mentioned in section 2 Thi.rd, the main sentence is identifjed by referring to the intersentential relations and the heading of the section under the main topics of the title. The lower level sentence is indented as a modifier of the main sentence. Sometimes, the knowledge of the specific field is required for better understanding of the relations among main sentential groups and various headings of the text. A case frame of a knowledge base for the specific field is provided in which each s.lot is filled with the most general term in the specific field. Fourth, a subframe name is prefixed to each main sentential group by reforring to the category of the main predicate term of the main sentence and the subframe designated by tho heading of the section and the titte of the text. The basic subframe names are, for example, FUNCTION, COMPOSJTION and PROPERTY in description of actions and physical objects.

As seen in the above, the main work of the text analysis is to identify the main sentential groups and to assign to them a standard attribute name of a subframe in a specified field. These frames and attribute names are used as a key of a specific field for efficiently storing and retrieving the knowledge contained in texts.

The next example of text analysis is taken from a technical paper in language processing. [Example 2]

Title: A natural language understanding system for data management

Heading of Section: Generating Finglish sentences Heading of Subsection: The selector

(1) The selector's main job is to construct: a graph relevant to the input statement. (2) In constructing this graph the selector first copies the portion of the semantic net which is to be output. (3) It then uses inverse mapping functions to produce a more surface, but still case graminar based representation of the information to be output. (4) Inverse mapping functions map the mumeric representation for date to a more surface one. (5)The selector constructs 
modality lisits next and chooses a surface ordering rule(SOR) for each verb of the resulting structure. (6)SORs specify the order of the syntactic cases associated to a particular verb to be output.

In the above text the intersentential relations and the levels of sentences are identified, and the label of a subframe is prefixed to each sentenco as shown in lig.2(a) and (b).

(1)

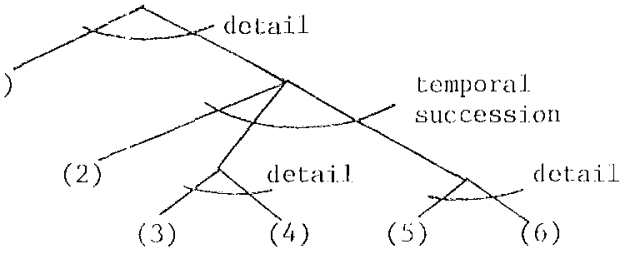

fig.2(a) The intersentential. relations

(1) FUNCIION; ( PREIJ) : construct, $\Lambda$ G: se lector, SUB-PROCHSS: OBJ $: \operatorname{graph}(\ldots \ldots)$,

(2) HUNCTION ; (PRED) : copy, $\Lambda G$ : se I.ector, OBJ : portion (...), MANN: f irst)

(3) FUNCI'TON ; (PRLD: produce, $\Lambda G$ : se $\perp$ ector , OBJ : moresurface...representation(....) , MANN : then, TNS'IR: inversemapping-functions

(4) (PREI): map, , AG:*, OBJ : numericropresentation (...), (...........)

(5) FUNCIION ; (PRED : construct, $\Lambda$ : se lector, OBJ : modal i ty-1.ists, MANN : next)

(PRLID) : choose, $A \mathrm{G}$ : selector, OBJ : surface-ordering-rule

(6) (PRLID: specif y, $A G$ : $:, O B J$ : $\operatorname{order}(\ldots)), \ldots \ldots))$

Fig.2(b) The composition of the text

A symbol. "sw" denotes a term profixed to thes subframe containing the mark "*" and modified by the subframe.

4. Generation of answering sentences for querios

Tn this section, sentence generelion or Lext generation for answering al request is described briefly. 'l'ext generation is the inverse process of Lext analysis and is inseparable from text analysis in a sense that the text generation provides an basic idea on text construction for given information to be represented. $A$ given query is parsed and the intermediate expression is constructed. J'hen the required information is retrieved and transformed into a surface expression in the following steps:

(1) The intermediate expressions related to the matin topics of the query are extracted in the order of the level rolated to the query from the analyzed text or the database storing i.t under a guide of: the frame labol and other heading information as well as the index of the terms contained in the text. The tevel of a description in the text is available for selection of the knowledge source to be extrated.

(2) The intermediate expressions are rearranged in the coherent and readable order, for example, in the occurrence order of the events, and an answer sequence is constructed.

(3) Under a given bounded length the answer. sequence is grouped or segmented to soveral parts and sentential topjcs are selected to be expanded into surface expressions.

(4) The sentential form of each of the scgments is selected to one of phrase, simple, complex and compousd surface expressions by referring to the sentential topic.

The summary of the text given in Example 2 is generated from the analyzed results shown in Fig.2(b) by referring to the steps 23 and 4. J'ig. 3 shows two sumaries constructed from the descriptions of the text up to level 1 and 3 , where the part cnclosed with brackets is the part: generated from the descriptions of level 3 .

level l:The selector constructs a graph relevant to the input statement.

level 3:The selector constructs a graph relevant to the input statement. In the construction, the selector performs the following processes. I'irst, the selector copies the portion of the scmantic net. Then, it: produces a more surface but case greminar based representation with inverse mapping funclions [which may) a numeri.c. representation to a more surface onel. linally, it constructs modality 1ists and chooses a surface ordering rule [ which specifies the order of syntactic cases ] for cach verb.

Fig.3 Generated sumaries

\section{Conclusion}

$\Lambda$ experimental. system is under construction bascd on our structured-information extraction system constructed previously. This paper focusses attention ort the content suggested by the heading and intersentential structures and assigns a sentence level to each sentence. Ellipsis and restoration problem of known structures on syntax and special field knowledge is not considered here. However, it seems that there are no serious problems in many specific fields at an interactive mode with users.

\section{References}

1) Rumcthart,D.J': Notes on a Schema for Storics, in Bobrow,D.G, and Collins, $\Lambda$. (eds.), Representation and Understanding, pp.211-236, Academic Pross, New York (1975).

2) Hobbs,J.R.: Coherence and Tnterpretation in Linglish Texts, Proc, 5th TJCAT, pp.110-116 (1977).

3) Kigney, J.W. and Munro, $\Lambda_{0}:$ On Cognitive Strategies for Processing Text, University of Southern California, Behavioral T'echnology laboratories, T'ech. Rep. No.80 (1977).

4) T'akanatsu, S., Fujita,Y. and Nishida,F.: Normalization of Titles and their Relrievel, Information Processing \& Managenent, Vol.16, pp. $155-167$ (1.980)

5) Nishida, $F$, and l'akamatsu, S.: StructuredInformation Extraction fron Patent-Claim Sentences, Information Processing \& Management, Vol.18, No.1, pp.1-13 (1982).

6) Nishida,F., Takametsu,S. and Iijita, Y.: Semiautonatic Indexing of Structured lnformation of Text, J. Chom. Inf. Comput. Sci., Vol.24, No.1, 1p.15-20 (19834). 